\title{
Simplified Bethe-Salpeter Description of Basic Pseudoscalar-Meson Features
}

\author{
Wolfgang Lucha ${ }^{1, *}$ \\ ${ }^{1}$ Institute for High Energy Physics, Austrian Academy of Sciences, Nikolsdorfergasse 18, \\ A-1050 Vienna, Austria
}

\begin{abstract}
We assess a description of pseudoscalar mesons as pseudo-Goldstone bosons by its compatibility with some Gell-Mann-Oakes-Renner-type relation.
\end{abstract}

\section{Pseudoscalar Mesons of Goldstone Nature}

Goldstone's theorem necessitates the presence of a massless boson in the spectrum of physical particles for any spontaneously broken chiral symmetry of quantum chromodynamics (QCD); these hypothetical Goldstone bosons are identified with the ground-state pseudoscalar mesons (pions, kaons, $\eta$ ). Their finite (but comparatively small) masses are attributed to the additional explicit breakdown of the chiral symmetries of QCD enforced by nonvanishing quark masses.

We analyze the Goldstone-boson nature of the lightest pseudoscalar mesons by means of a formalism [1-4] situated somewhere between the fully relativistic Bethe-Salpeter description of bound states [5], with several still to be resolved inherent obstacles, and the latter's extreme instantaneous limit, represented by its three-dimensional reduction devised by Salpeter [6]. A rather promising tool to judge the merits of such kind of intermediate framework proves to be, among others, the fulfilment of (generalized) Gell-Mann-Oakes-Renner-type relations [7] by characteristic features (viz., decay constants or condensates) of light pseudoscalar mesons [8].

\section{Quark-Antiquark Bound-State Formalism}

The homogeneous Bethe-Salpeter equation describes, in Poincaré-covariant manner, a bound state $|\mathrm{B}(P)\rangle$ of mass $\widehat{M}$ and momentum $P$, formed by two particles of relative momentum $p$, by its Bethe-Salpeter amplitude $\Phi(p, P)$. One ingredient are the constituents' full propagators, in the case of spin- $\frac{1}{2}$ fermions $i$ given by mass $M_{i}\left(p^{2}\right)$ and wave-function renormalization $Z_{i}\left(p^{2}\right)$ :

$$
S_{i}(p)=\frac{\mathrm{i} Z_{i}\left(p^{2}\right)}{\not p-M_{i}\left(p^{2}\right)+\mathrm{i} \varepsilon}, \quad \not p \equiv p^{\mu} \gamma_{\mu}, \quad \varepsilon \downarrow 0, \quad i=1,2 .
$$

The other ingredient are the interactions responsible for bound-state formation. Ignoring their dependence on time components of momenta and the above propagators' dependence on zero momentum components squared led to a bound-state equation [1] for a Salpeter amplitude [6]

$$
\phi(\boldsymbol{p}) \propto \int \mathrm{d} p_{0} \Phi(p, P),
$$

interpretable as equal-time bound-state wave function. An integral kernel $K(\boldsymbol{p}, \boldsymbol{q})$ captures the entirety of, by assumption instantaneous, effective interactions of all bound-state constituents.

\footnotetext{
*e-mail: Wolfgang.Lucha@oeaw.ac.at
} 
The bound-state equation proposed in Ref. [1], formulated in terms of the kinetic energies,

$$
E_{i}(\boldsymbol{p}) \equiv \sqrt{\boldsymbol{p}^{2}+M_{i}^{2}\left(\boldsymbol{p}^{2}\right)}
$$

and the energy projectors onto positive and negative energies of the bound-state constituents $i$,

$$
\Lambda_{i}^{ \pm}(\boldsymbol{p}) \equiv \frac{E_{i}(\boldsymbol{p}) \pm \gamma_{0}\left[\boldsymbol{\gamma} \cdot \boldsymbol{p}+M_{i}\left(\boldsymbol{p}^{2}\right)\right]}{2 E_{i}(\boldsymbol{p})}
$$

reads, for the case of fermion-antifermion bound states in the center-of-momentum frame [1],

$$
\begin{aligned}
\phi(\boldsymbol{p})=Z_{1}\left(\boldsymbol{p}^{2}\right) Z_{2}\left(\boldsymbol{p}^{2}\right) \int \frac{\mathrm{d}^{3} q}{(2 \pi)^{3}} & \left(\frac{\Lambda_{1}^{+}(\boldsymbol{p}) \gamma_{0}[K(\boldsymbol{p}, \boldsymbol{q}) \phi(\boldsymbol{q})] \Lambda_{2}^{-}(\boldsymbol{p}) \gamma_{0}}{\widehat{M}-E_{1}(\boldsymbol{p})-E_{2}(\boldsymbol{p})}\right. \\
- & \left.\frac{\Lambda_{1}^{-}(\boldsymbol{p}) \gamma_{0}[K(\boldsymbol{p}, \boldsymbol{q}) \phi(\boldsymbol{q})] \Lambda_{2}^{+}(\boldsymbol{p}) \gamma_{0}}{\widehat{M}+E_{1}(\boldsymbol{p})+E_{2}(\boldsymbol{p})}\right) .
\end{aligned}
$$

For any one-particle states $|B(P)\rangle$ normalized according to the Lorentz-invariant condition

$$
\left\langle B(P) \mid B\left(P^{\prime}\right)\right\rangle=(2 \pi)^{3} 2 P_{0} \delta^{(3)}\left(\boldsymbol{P}-\boldsymbol{P}^{\prime}\right),
$$

the normalization condition for the corresponding Salpeter amplitudes $\phi(\boldsymbol{p})$ is given by [9-11]

$$
\int \frac{\mathrm{d}^{3} p}{(2 \pi)^{3}} \operatorname{Tr}\left[\phi^{\dagger}(\boldsymbol{p}) \frac{\gamma_{0}\left[\boldsymbol{\gamma} \cdot \boldsymbol{p}+M_{1}\left(\boldsymbol{p}^{2}\right)\right]}{E_{1}(\boldsymbol{p})} \phi(\boldsymbol{p})\right]=2 P_{0} .
$$

\section{Assuming Generalized Flavour Symmetry}

Things simplify considerably if the propagator functions of involved fermion and antifermion happen to be identical, $M_{1}\left(\boldsymbol{p}^{2}\right)=M_{2}\left(\boldsymbol{p}^{2}\right)$ and $Z_{1}\left(\boldsymbol{p}^{2}\right)=Z_{2}\left(\boldsymbol{p}^{2}\right)$; the Salpeter amplitude of every pseudoscalar bound state is then fully defined by just two Lorentz-scalar components, $\varphi_{1,2}(\boldsymbol{p})$ :

$$
\phi(\boldsymbol{p})=\frac{1}{\sqrt{3}}\left[\varphi_{1}(\boldsymbol{p}) \frac{\gamma_{0}\left[\boldsymbol{\gamma} \cdot \boldsymbol{p}+M\left(\boldsymbol{p}^{2}\right)\right]}{E(\boldsymbol{p})}+\varphi_{2}(\boldsymbol{p})\right] \gamma_{5} .
$$

For $K(\boldsymbol{p}, \boldsymbol{q})$ compatible with spherical and rather specific Fierz symmetries of the effective interactions, our bound-state equation governing $\phi(\boldsymbol{p})$ collapses to an eigenvalue problem [12] fixing the radial parts $\varphi_{1,2}(p), p \equiv|\boldsymbol{p}|$, of $\varphi_{1,2}(\boldsymbol{p})$, with a single, spherically symmetric potential $V(r), r \equiv|\boldsymbol{x}|$, encoding the configuration-space interactions between bound-state constituents:

$$
\begin{aligned}
& E(p) \varphi_{2}(p)+\frac{2 Z^{2}\left(p^{2}\right)}{\pi p} \int_{0}^{\infty} \mathrm{d} q q \mathrm{~d} r \sin (p r) \sin (q r) V(r) \varphi_{2}(q)=\frac{\widehat{M}}{2} \varphi_{1}(p), \\
& E(p) \varphi_{1}(p)=\frac{\widehat{M}}{2} \varphi_{2}(p) .
\end{aligned}
$$

For the actual case of interest, mesonic bound states of quarks and antiquarks, the effective interaction potential $V(r)$ was extracted pointwise [2-4] by straightforward inversion $[13,14]$ of our Bethe-Salpeter-inspired bound-state equation [1], starting from that Salpeter amplitude $\phi(p)$ that represents massless pseudoscalar mesons. The latter, in turn, is connected [15-17] to the chiral-quark propagator [18, 19] by a Ward-Takahashi identity of QCD [7]. The emerging confining $V(r)$ rises, from its slightly negative value at $r=0$, rather steeply to infinity (Fig. 1). 


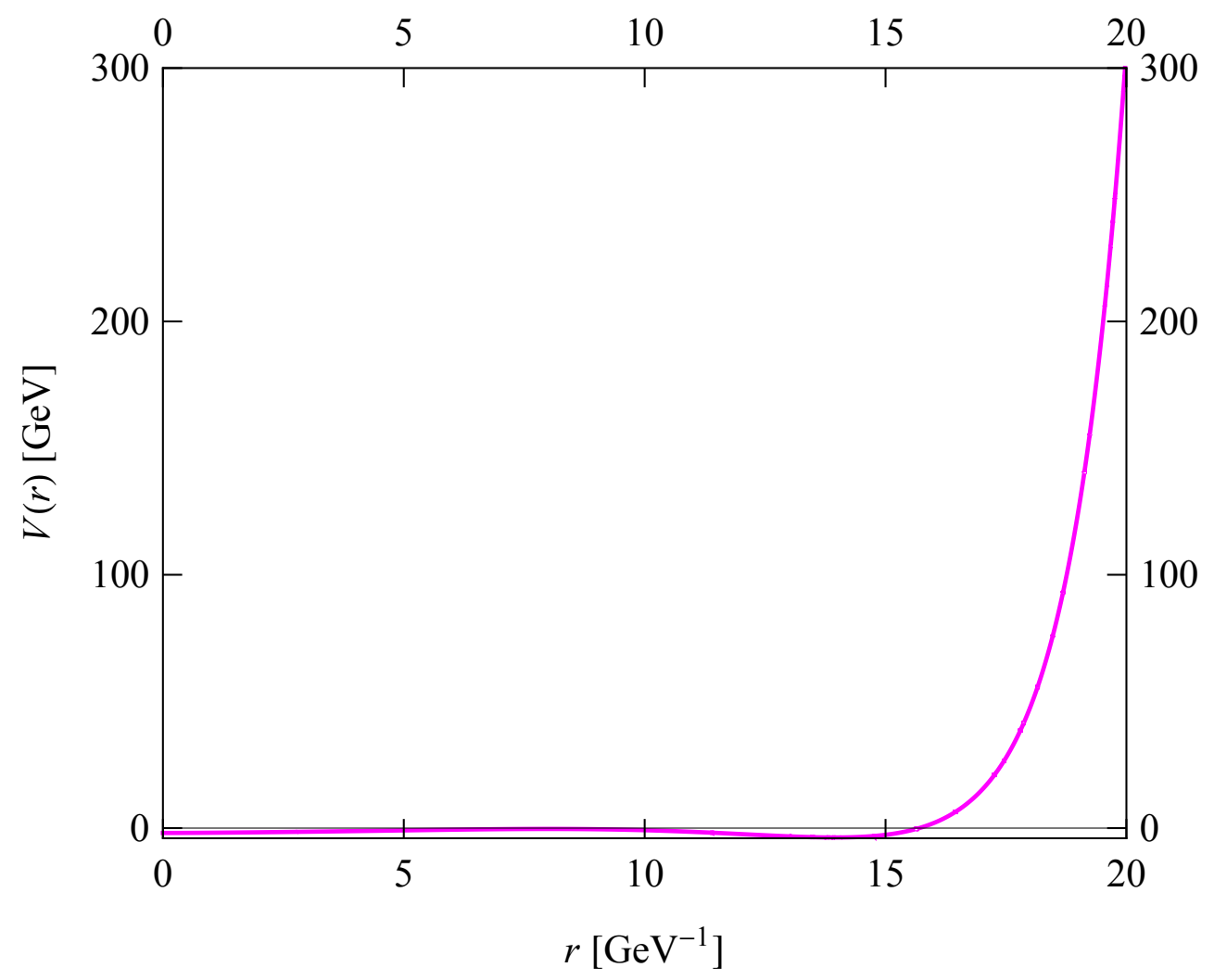

Figure 1. Effective interquark central potential $V(r)$ pinned down by inverting the radial Bethe-Salpeter problem (1) for a Salpeter-amplitude input derived from some quark-propagator model solution $[18,19]$. The almost flatness of the potential $V(r)$ near the origin in combination with its extraordinarily steep rise to infinity renders the form of $V(r)$ pretty close but clearly not identical to that of a square-well potential.

\section{Pseudoscalar-Meson Properties Revisited}

In order to scrutinize pseudoscalar quark-antiquark bound states for physical (i.e., non-chiral) quark masses, we ought to find the corresponding solutions to our set (1) of coupled equations for the two independent radial Salpeter components $\varphi_{1,2}(p)$. Our task is greatly facilitated by a definitely obvious move enabled by the purely algebraic nature of one of the two relations (1): inserting any of the two relations (1) into the other leads to single explicit eigenvalue problems for either $\varphi_{1}(p)$ or $\varphi_{2}(p)$ with mass-squared eigenvalue $\widehat{M}^{2}[2-4,12]$; conversion to equivalent matrix eigenvalue problems by expansion over a convenient function-space basis is among the standard solution procedures [20-24]. With these solutions at hand, we may create trust in the reliability of our approach by assessing and exploring its predictions for hadronic observables.

The spatial extension of the pion deduced, from the numerical ground-state solution to our bound-state formalism inferred along the above lines, in form of the pion's average interquark distance $\langle r\rangle=0.478 \mathrm{fm}$ or root-mean-square radius $\sqrt{\left\langle r^{2}\right\rangle}=0.529 \mathrm{fm}$ fits nicely to the pion's electromagnetic charge radius, $\sqrt{\left\langle r_{\pi}^{2}\right\rangle}=(0.672 \pm 0.008) \mathrm{fm}$ [25]. Nevertheless, this agreement won't qualify as confirmation of the credibility of our framework since the Salpeter amplitude for chiral quarks served already as input to the inversion procedure that generated the shape of our potential $V(r)$, and the experimental $u / d$ quark masses are pretty close to their chiral limit. 
Consequently, we have to look for different and more significant criteria which allow us to appraise the reasonableness of any inversion considerations. Fortunately, this proves to be not too difficult: Equating the residues of pseudoscalar-meson pole terms in both axial-vector and pseudoscalar vertex functions entering into the axial-vector Ward-Takahashi identity of QCD expressing the invariance of QCD under chiral transformations leads to a generalization [7] of the Gell-Mann-Oakes-Renner relation [26]; this innovation relates, for a pseudoscalar bound state $|B(P)\rangle$, its (weak) decay constant $f_{B}$, defined in terms of the axial-vector quark current by

$$
\left\langle 0\left|: \bar{\psi}_{1}(0) \gamma_{\mu} \gamma_{5} \psi_{2}(0):\right| B(P)\right\rangle=\mathrm{i} f_{B} P_{\mu},
$$

— which, consequently, may be found by projection of $\phi(\boldsymbol{p})$ onto the axial-vector current, i.e.,

$$
f_{B} \propto \int \mathrm{d}^{3} p \operatorname{Tr}\left[\gamma_{0} \gamma_{5} \phi(\boldsymbol{p})\right]
$$

— and its in-hadron condensate [7] (universalizing the notion of quark vacuum condensates) ${ }^{1}$

$$
\mathbb{C}_{B} \equiv\left\langle 0\left|: \bar{\psi}_{1}(0) \gamma_{5} \psi_{2}(0):\right| B(P)\right\rangle \propto \int \mathrm{d}^{3} p \operatorname{Tr}\left[\gamma_{5} \phi(\boldsymbol{p})\right]
$$

to the mass $\widehat{M}_{B}$ of this pseudoscalar bound state and the two relevant quark mass parameters in the QCD Lagrangian [7]. For the simpler case of equal quark masses $m$, this relationship reads

$$
f_{B} \widehat{M}_{B}^{2}=2 m \mathbb{C}_{B} \text {. }
$$

Compatibility with Eq. (2) may be inspected by solving our formalism with the previously established potential $V(r)$ for bound states of chiral, $u / d$, and $s$ quarks, taking advantage of the appropriate model propagator functions $[18,19]$. Comparison of the quark masses $m$, fixed by the thus determined values of $\widehat{M}_{B}, f_{B}$, and $\mathbb{C}_{B}$, with the current-quark masses $\bar{m}(\mu)$ in modified minimal subtraction at scale $\mu$ proves that our findings for $m$ are in the right ballpark (Table 1).

Table 1. Predictions of the Bethe-Salpeter-inspired bound-state equation (1), with effective interaction potential $V(r)$ as depicted in Fig. 1 , for masses $\widehat{M}_{B}$, decay constants $f_{B}$ and in-meson condensates $\mathbb{C}_{B}$ of the lightest pseudoscalar mesons, and confrontation of the quark-mass parameters $m$ resulting from the more general Gell-Mann-Oakes-Renner relation (2) with their minimal-subtraction counterparts $\bar{m}(\mu)$.

\begin{tabular}{rrcccc}
\hline Constituents & $\begin{array}{c}\widehat{M}_{B} \\
{[\mathrm{MeV}]}\end{array}$ & $\begin{array}{c}f_{B} \\
{[\mathrm{MeV}]}\end{array}$ & $\begin{array}{c}\mathbb{C}_{B} \\
{\left[\mathrm{GeV}^{2}\right]}\end{array}$ & $\begin{array}{c}m \\
{[\mathrm{MeV}]}\end{array}$ & $\begin{array}{c}\bar{m}(2 \mathrm{GeV}) \\
{[\mathrm{MeV}][25]}\end{array}$ \\
\hline chiral quarks & 6.8 & 151 & 0.585 & 0.0059 & - \\
$u / d$ quarks & 148.6 & 155 & 0.598 & 2.85 & $3.5_{-0.3}^{+0.7}$ \\
$s$ quarks & 620.7 & 211 & 0.799 & 51.0 & $96_{-4}^{+8}$ \\
\hline
\end{tabular}

\section{Summary of Findings and Conclusions}

In order to establish whether or not it is justified to lend trust to the outcomes of an approach to bound states proposed some time ago [1] and residing, as far as its compatibility with Poincaré covariance is concerned, somewhere in the vast range between bound-state descriptions along the ideas of Salpeter and Bethe, on the one hand, and static approximations, on the other hand, we evaluated the performance of the investigated framework's predictions for those properties of the lightest pseudoscalar mesons that happen to be related by an advancement of the insight gained by Gell-Mann, Oakes, and Renner [26]: In brief, the formalism of Ref. [1] is still alive.

\footnotetext{
${ }^{1}$ A recent, comprehensive, Bethe-Salpeter-rooted evaluation of in-hadron condensates may be found in Ref. [27].
} 


\section{References}

[1] W. Lucha and F. F. Schöberl, J. Phys. G 31 (2005) 1133, arXiv:hep-th/0507281.

[2] W. Lucha and F. F. Schöberl, Int. J. Mod. Phys. A 31 (2016) 1650202, arXiv:1606.04781 [hep-ph].

[3] W. Lucha, EPJ Web Conf. 129 (2016) 00047, arXiv:1607.02426 [hep-ph].

[4] W. Lucha, EPJ Web Conf. 137 (2017) 13009, arXiv:1609.01474 [hep-ph].

[5] E. E. Salpeter and H. A. Bethe, Phys. Rev. 84 (1951) 1232.

[6] E. E. Salpeter, Phys. Rev. 87 (1952) 328.

[7] P. Maris, C. D. Roberts, and P. C. Tandy, Phys. Lett. B 420 (1998) 267, arXiv:nucl-th/ 9707003.

[8] W. Lucha and F. F. Schöberl, Int. J. Mod. Phys. A 33 (2018) 1850047, arXiv:1801.00264 [hep-ph].

[9] J.-F. Lagaë, Phys. Rev. D 45 (1992) 305.

[10] J. Resag, C. R. Münz, B. C. Metsch, and H. R. Petry, Nucl. Phys. A 578 (1994) 397, arXiv:nucl-th/9307026.

[11] M. G. Olsson, S. Veseli, and K. Williams, Phys. Rev. D 52 (1995) 5141, arXiv:hep-ph/ 9503477.

[12] Z.-F. Li, W. Lucha, and F. F. Schöberl, Phys. Rev. D 76 (2007) 125028, arXiv:0707.3202 [hep-ph].

[13] W. Lucha and F. F. Schöberl, Phys. Rev. D 87 (2013) 016009, arXiv:1211.4716 [hep$\mathrm{ph}]$.

[14] W. Lucha, Proc. Sci., EPS-HEP 2013 (2013) 007, arXiv:1308.3130 [hep-ph].

[15] W. Lucha and F. F. Schöberl, Phys. Rev. D 92 (2015) 076005, arXiv:1508.02951 [hep$\mathrm{ph}]$.

[16] W. Lucha and F. F. Schöberl, Phys. Rev. D 93 (2016) 056006, arXiv:1602.02356 [hep$\mathrm{ph}$.

[17] W. Lucha and F. F. Schöberl, Phys. Rev. D 93 (2016) 096005, arXiv:1603.08745 [hep$\mathrm{ph}$.

[18] P. Maris and P. C. Tandy, Phys. Rev. C 60 (1999) 055214, arXiv:nucl-th/9905056.

[19] P. Maris, in Proceedings of the International Conference on Quark Confinement and the Hadron Spectrum IV, editors W. Lucha and K. Maung Maung (World Scientific, Singapore, 2002), p. 163, arXiv:nucl-th/0009064.

[20] W. Lucha and F. F. Schöberl, Phys. Rev. A 56 (1997) 139, arXiv:hep-ph/9609322.

[21] W. Lucha and F. F. Schöberl, Int. J. Mod. Phys. A 14 (1999) 2309, arXiv:hep-ph/ 9812368.

[22] W. Lucha, K. Maung Maung, and F. F. Schöberl, Phys. Rev. D 63 (2001) 056002, arXiv:hep-ph/0009185.

[23] W. Lucha, K. Maung Maung, and F. F. Schöberl, Phys. Rev. D 64 (2001) 036007, arXiv:hep-ph/0011235.

[24] W. Lucha and F. F. Schöberl, Recent Res. Dev. Phys. 5 (2004) 1423, arXiv:hep-ph/ 0408184.

[25] C. Patrignani et al. (Particle Data Group), Chin. Phys. C 40 (2016) 100001.

[26] M. Gell-Mann, R. J. Oakes, and B. Renner, Phys. Rev. 175 (1968) 2195.

[27] T. Hilger, M. Gómez-Rocha, A. Krassnigg, and W. Lucha, Eur. Phys. J. A 53 (2017) 213, arXiv:1702.06262 [hep-ph]. 